\title{
Processos de difusão de partículas da mesma natureza em dois estados de energia diferentes
}

Particles of the same nature diffusing in two distinct energy states

\author{
Luiz Bevilacqua* ${ }^{* 10}$
}

\author{
${ }^{1}$ Universidade Federal do Rio de Janeiro, Instituto Alberto Luiz Coimbra de Pós-Graduação e Pesquisa de Engenharia, \\ Rio de Janeiro, RJ, Brasil.
}

\begin{abstract}
Recebido em 24 de agosto de 2021. Revisado em 28 de setembro de 2021. Aceito em 30 de setembro de 2021.
O texto a seguir trata de processos de difusão de partículas de mesma natureza divididas em dois conjuntos complementares que se deslocam estimuladas por dois potenciais distintos. O fluxo principal obedece à lei clássica de Fick e o fluxo secundário é subsidiário ao principal e sujeito a uma nova lei. A teoria é particularmente útil para processos em que as partículas representam variáveis que podem mudar de estado como é o caso em fluxo de capitais e na dinâmica populacional.
\end{abstract}

Palavras-chave: Difusão, fluxo de capitais, modelos discretos, equações de quarta ordem, difusão anômala.

The present text deals with the diffusion process of particles of the same nature split into two distinct sets scattering subjected to the action of two distinct potentials. The main flux follows Fick's law and the secondary flux which is subsidiary to the main flux follows a new law. The theory is particularly important for analyzing the diffusion of particles that can change energy states. Capital flux and population dynamics are cases where the theory can find important applications.

Keywords: Diffusion, capital flux, discrete models, fourth order equations, anomalous diffusion.

\section{Introdução}

O espalhamento ordenado de partículas em um determinado meio é denominado de difusão. A difusão pode ocorrer em um grande espectro de fenômenos. De fato, a difusão prevalece não apenas em fenômenos físicoquímicos, que são importantes em processos industriais e fenômenos naturais, mas também em dinâmica populacional, propagação de notícias e desenvolvimentos socioeconômicos, para dar alguns exemplos. A regularidade do processo de difusão permite que seja representado analiticamente, isto é, admite a descrição com modelos matemático-computacionais.

Como as primeiras observações experimentais que suscitaram o desenvolvimento do modelo matemático focalizaram um processo de espalhamento a teoria foi orientada para a descrição de um fenômeno de desconcentração de conteúdo. Se o processo observado inicialmente fosse o movimento de micro-organismos aproximando-se de uma fonte de alimentos, talvez a teoria tivesse tomado outro rumo.

Antes de iniciar a apresentação da modelagem da difusão é conveniente definir o que se entende por fluxo de partículas que é o fenômeno básico subjacente ao processo. Considere um conjunto de partículas que se dispersa ao longo de uma direção preferencial à qual

\footnotetext{
*Endereço de correspondência: bevilacqua@coc.ufrj.br
}

chamaremos de x. Admite-se que a distribuição das partículas seja uniforme na área ortogonal ao movimento, de modo que a densidade de massa é $q(x, t)=$ $\lim _{\Delta x, \Delta m \rightarrow 0}(\Delta m / S \Delta x)$, onde $S$ é a secção transversal ao fluxo sobre a qual se distribuem as partículas e $\Delta m$ a massa no volume elementar $\Delta V=S \Delta x$. O fluxo é definido como $\boldsymbol{\Psi}(x, t)=\lim _{\Delta m, \Delta t \rightarrow 0}(\Delta m / S \Delta t) \mathbf{n}_{1}$, onde $\mathbf{n}_{1}$ é o vetor unitário na direção $\mathrm{x}$, Fig. 1 .

Para o desenvolvimento que se segue é conveniente definir uma grandeza auxiliar que chamaremos de potencial de fluxo ou simplesmente potencial. O potencial é definido como uma função continua $\hat{P}=\hat{P}(x)$, suficientemente diferenciável, tal que o fluxo seja dado por:

$$
\boldsymbol{\Psi}=-K \frac{\partial \hat{P}}{\partial x} \mathbf{n}_{1}
$$

Onde $K$ é uma constante e $\mathbf{n}_{1}$ o vetor unitário na direção x. No caso clássico de difusão temos $\hat{P}=q(x, t)$ que é a própria distribuição de densidade e $K$ uma

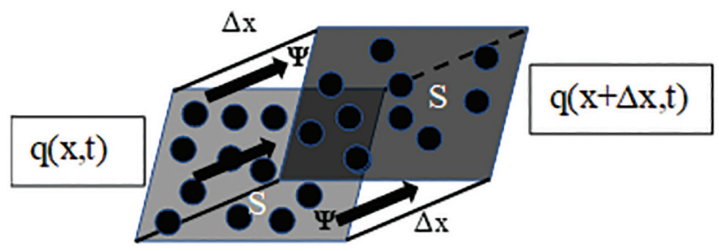

Figura 1: Fluxo de partículas na caixa $\mathrm{S} \Delta \mathrm{x}$. 
constante designada comumente com a letra $D$. Foi A. Fick [1] que no fim do século XIX propôs essa lei de difusão a partir de cuidadosas experiências que foram motivadas pelas observações de Brown [2]. Então o fluxo para processos clássicos ou processos de Fick é definido como:

$$
\boldsymbol{\Psi}_{1}=-D \frac{\partial q}{\partial x} \mathbf{n}_{1}
$$

Este trabalho introduz a presença de um fluxo paralelo coexistente com o fluxo clássico de Fick. Admite-se que um subconjunto de partículas possa ser transferido do processo de Fick para um processo paralelo secundário sensível a um potencial diferente do potencial clássico $\hat{P}=q(x, t)$ apresentado acima. O fenômeno consiste, portanto, de dois fluxos paralelos constituídos por dois conjuntos de partículas ativados por dois potenciais distintos. Consequentemente o processo global evolui em dois microestados que se espalham segundo potenciais de ativação diferentes. O estado principal obedece à lei de Fick e o estado subsidiário dispersa segundo uma lei diferente da lei de Fick. Portanto, é conveniente, e muitas vezes necessário, admitir que partículas participando do processo global possam migrar de um fluxo para outro. Isto é, além do movimento induzido pelo processo de difusão pode existir um outro movimento provocando a troca de estado das partículas que migram de um fluxo para outro. Dois exemplos típicos ilustram a necessidade de adoção de um modelo que considere a existência de dois fluxos com troca de estado. O primeiro considera o movimento de populações e o segundo simula a trajetória de capital em um meio economicamente ativo.

O primeiro exemplo é o caso do fluxo de indivíduos pacientes entrando e recuperados saído - em um hospital. Isto é, considerar o movimento de pessoas infectadas que circulam simultaneamente com os recuperados em um mesmo ambiente. Os infectados podem mudar de estado passando para recuperados, o que parece ser o movimento mais importante, embora também possa ocorrer a transferência inversa que é a passagem de recuperados para infectados o que também pode ser levado em conta no modelo que vamos propor. Certamente é ainda necessário considerar a ocorrência de óbitos o que não é difícil de ser feito nas equações representativas do fenômeno. Os modelos clássicos não dão conta desse processo.

O segundo caso trata de fluxo de capitais. Para ilustrar podemos pensar no comportamento de alguém que recebe o salário mensal e o gasta para viver. $\mathrm{O}$ dinheiro que entra tende a um estado de acumulação, mas logo esse mesmo dinheiro é transferido para outro estado, o de gasto, destinado a compra dos meios necessários para viver. Tanto para economia doméstica como para pequenos negócios, ou mesmo para empresas relativamente grande, o fluxo de capital tem duas direções, sendo, no entanto, composto pelo mesmo numerário que é acumulado como poupança ou como gasto na despesa necessária para manter o negócio ativo. Na realidade a evolução do fluxo de capitais foi modelada por Black e Scholes [3], dois professores da Universidade de Chicago, como um processo de difusão admitindo a teoria clássica de Fick e consequentemente movimento em apenas uma direção. Certamente este é um fenômeno que admite, e até mesmo requer, a consideração de fluxo duplo conforme proposto acima.

A teoria que vamos apresentar a seguir considera a evolução de dois processos de difusão em paralelo, cada processo sujeito a um potencial de fluxo específico sendo que as partículas podem transitar entre as duas correntes de difusão.

\section{Processos Difusivos. Fluxo Simples}

São vários os métodos de derivação da equação da difusão. As formulações mais recentes recorrem à instituição de uma equação mestra (master equation), que descreve a evolução no tempo das variáveis de estado segundo trajetórias probabilísticas. Alternativamente, formulações discretas, que normalmente são limitadas a processos bem determinados, podem conduzir a resultados que revelam fenômenos não previstos como veremos adiante. Inicialmente vamos tratar do caso clássico de difusão que se desenvolve segundo uma direção bem determinada. Podemos admitir que as partículas depositadas em uma célula $\mathrm{n}$ em um tempo $\mathrm{t}$ são transferidas em partes iguais para as células vizinhas $n+1$ e $n-1$ no intervalo de tempo $\Delta$ t Fig. 2. As equações que descrevem esse processo são obtidas sem dificuldade, Eqs. 3a e $(3 b)$ :

$$
\begin{aligned}
q_{n}^{t} & =\frac{1}{2} q_{n-1}^{t-\Delta t}+\frac{1}{2} q_{n+1}^{t-\Delta t} \\
q_{n}^{t+\Delta t} & =\frac{1}{2} q_{n-1}^{t}+\frac{1}{2} q_{n+1}^{t}
\end{aligned}
$$

Fazendo a diferença $\left(q_{n}^{t+\Delta t}-q_{n}^{t}\right)$ obtém-se:

$$
q_{n}^{t+\Delta t}-q_{n}^{t}=\frac{1}{2}\left(q_{n-1}^{t}+q_{n+1}^{t}-q_{n-1}^{t-\Delta t}-q_{n+1}^{t-\Delta t}\right)
$$

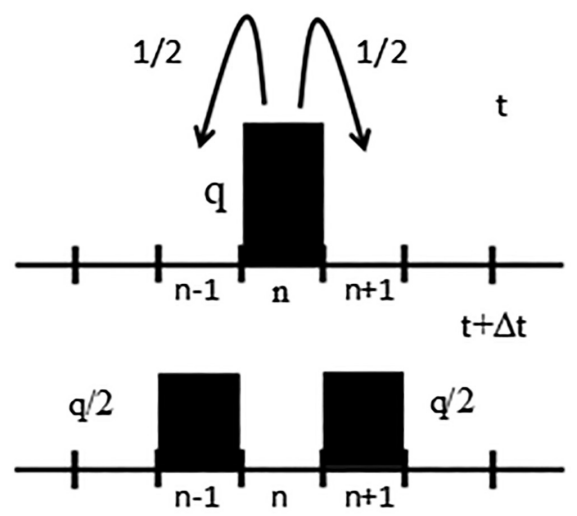

Figura 2: Redistribuição simétrica sem retenção. 
É conveniente reduzir todos os termos à direita da Eq. (4) ao tempo $(t-\Delta t)$. Observando que:

$$
\begin{aligned}
& q_{n-1}^{t}=\frac{1}{2} q_{n-2}^{t-\Delta t}+\frac{1}{2} q_{n}^{t-\Delta t} \\
& q_{n+1}^{t}=\frac{1}{2} q_{n}^{t-\Delta t}+\frac{1}{2} q_{n+2}^{t-\Delta t}
\end{aligned}
$$

e substituindo em (4) obtém-se:

$$
\begin{aligned}
q_{n}^{t+\Delta t}-q_{n}^{t}= & \frac{1}{4}\left[\left(q_{n-2}-2 q_{n-1}+q_{n}\right)\right. \\
& \left.+\left(q_{n+2}-2 q_{n+1}+q_{n}\right)\right]^{t-\Delta t}
\end{aligned}
$$

O termo à direita na equação (5) é a aproximação discreta de dois diferenciais de segunda ordem e o termo à esquerda a aproximação de um diferencial de primeira ordem (ver Apêndice I). Portanto podemos escrever como aproximação discreta da equação (5):

$$
\Delta q_{n}^{t+\Delta t}=\frac{1}{4}\left\{\Delta^{2} q_{n-1}+\Delta^{2} q_{n+1}\right\}^{t-\Delta t}
$$

Onde temos à esquerda a diferença de primeira ordem em relação a t e à direita diferenças de segunda ordem em relação a x. Fazendo-se as relações correspondentes a essas diferenças observando as respectivas ordens, isto é, com os intervalos $\Delta t$ para a variação no tempo, à esquerda, e $\Delta \mathrm{x}^{2}$ para a variação no espaço, à direita, vem:

$$
\frac{\Delta q_{n}^{t+\Delta t}}{\Delta t}=\frac{1}{4} \frac{\Delta x^{2}}{\Delta t}\left\{\frac{\Delta^{2} q_{n-1}}{\Delta x^{2}}+\frac{\Delta^{2} q_{n+1}}{\Delta x^{2}}\right\}^{t-\Delta t}
$$

Admitindo-se a função $q(x, t)$ suficientemente suave as diferenças correspondentes aos pontos $n+1$ e $n-1$ afastam-se da diferença correspondente ao ponto $\mathrm{n}$ por termos de ordem superior a $\Delta x^{2}$ de modo que podemos escrever:

$$
\frac{\Delta q_{n}^{t+\Delta t}}{\Delta t}=\frac{1}{2} \frac{\Delta x^{2}}{\Delta t}\left\{\frac{\Delta^{2} q_{n}}{\Delta x^{2}}+O\left(\Delta x^{2}\right)\right\}^{t-\Delta t}
$$

Tomando-se os limites para $\Delta x \rightarrow 0, \Delta t \rightarrow 0$ e $\Delta q_{n} \rightarrow$ 0 e com as notações $\left(\Delta x^{2} / \Delta t\right) \rightarrow 2 D, \Delta q_{n} / \Delta t \rightarrow \partial q_{n} / \partial t$ e $\Delta^{2} q_{n} / \Delta x^{2} \rightarrow \partial^{2} q_{n} / \partial x^{2}$ e ainda notando que com a hipótese de regularidade admitida acima $O\left(\Delta x^{2}\right) \rightarrow 0$ obtém-se:

$$
\frac{\partial q}{\partial t}=D \frac{\partial^{2} q}{\partial x^{2}}
$$

que é a equação clássica para difusão de partículas inertes em meios homogêneos. Note que a relação $\Delta x^{2} / \Delta t$ na definição do coeficiente de difusão $D$ é admitida finita, diferente de zero. Esta relação é uma particularidade que aparece na derivação da equação de difusão usando a formulação discreta. Einstein 4 mostrou que o deslocamento da posição média do conjunto de partículas varia $\mathrm{q}(\mathrm{x}, \mathrm{t})$

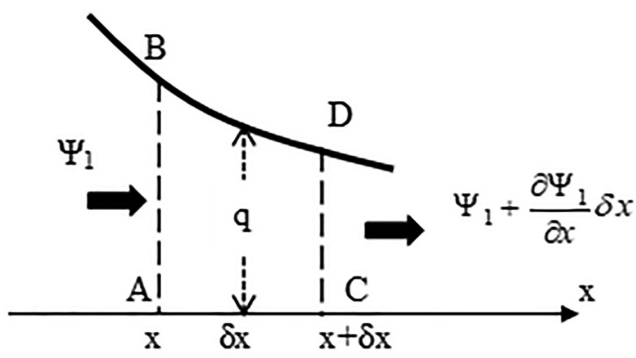

Figura 3: Fluxo de partículas ao longo do eixo $x . q(x, t)$ representa a densidade de partículas na secção $x$ e no tempo t e $\Psi_{1}$ o respectivo fluxo de partículas.

com a raiz quadrada do tempo, $\Delta x \sim \Delta t^{1 / 2}$ conferindo significado à relação $\Delta x^{2} / \Delta t$ que assume valores finitos e diferentes de zero para os limites $\Delta x \rightarrow 0, \Delta t \rightarrow 0$.

Agora, como o fenômeno correspondente à equação (8) está relacionado a transporte de partículas, a sua derivação pode ser feita a partir do principio de conservação de massa, desde que seja conhecida a expressão para os respectivos fluxos. Considere a distribuição de densidade de massa da Fig. 3

$\mathrm{Na}$ secção correspondente ao ponto $x$ o fluxo de massa relativo às partículas em movimento é dado por $\boldsymbol{\Psi}_{1} S$ e na secção correspondente ao ponto $x+\delta x$ por $\left[\boldsymbol{\Psi}_{1}+\left(\partial \boldsymbol{\Psi}_{1} / \partial x\right) \delta x\right] S$ onde $\boldsymbol{\Psi}_{1}$ é o vetor fluxo $\boldsymbol{\Psi}_{1}=\Psi_{1} \mathbf{n}_{1}$, sendo $\mathbf{n}_{1}$ o vetor unitário na direção x e $\Psi_{1}$ o módulo do vetor fluxo $\Psi_{1}=\left|\boldsymbol{\Psi}_{1}\right|$. A secção transvesal $\mathrm{S}$ é constantte no presente caso que trata de um processo homogêneo.

A massa correspondente ao volume de partículas no espaço ABCD é dado por $q S \delta x$ no instante $t$. Após um determinado intervalo de tempo $\delta t$ a massa será igual a $(q+(\partial q / \partial t) \delta t) S \delta x$. Portanto a variação de massa no volume $\mathrm{ABCD}=\Delta \mathrm{M}$ no intervalo $\delta t$ será:

$$
\Delta M_{1}=\int_{V}\left[q+\frac{\partial q}{\partial t} \delta t-q\right] S \delta x
$$

Mas essa variação de massa deve ser igual à diferença entre as massas correspondentes ao volume de partículas que entram em $\mathrm{ABCD}$ pela secção $\mathrm{AB}$ no intervalo de tempo $\delta t$, igual a $\Psi_{1}(x, t) S \delta t$, e as que saem pela secção CD no mesmo intervalo de tempo, igual a $\left[\Psi_{1}+\right.$ $\left.\left(\partial \Psi_{1} / \partial x\right) \delta x\right] S \delta t$. Podemos operar com o módulo de $\boldsymbol{\Psi}_{1}$ porque no nosso caso o fluxo é paralelo ao eixo $\mathrm{x}$. A variação de massa em $\mathrm{ABCD}$ pode ser calculada a partir da diferença entre as massas correspondentes aos volumes de partículas que entram e das partículas que saem. Com as definições acima essa diferença é dada por:

$$
\Delta M_{2}=\int_{V}\left[\Psi_{1}-\left(\Psi_{1}+\frac{\partial \Psi_{1}}{\partial x} \delta x\right)\right] S \delta t
$$


O princípio de conservação de massa impõe que $\Delta M_{1}-$ $\Delta M_{2}=0$ donde se segue com (9) e (10):

$$
\begin{aligned}
\Delta M & =\left(\Delta M_{1}-\Delta M_{2}\right) \\
& =\left\{\int_{V}\left(\frac{\partial q}{\partial t}+\frac{\partial \Psi_{1}}{\partial x}\right) S \delta x\right\} \delta t=0
\end{aligned}
$$

para qualquer $\Delta M$, isto é, para qualquer região em que se processa a difusão. Portanto é necessário que:

$$
\frac{\partial q}{\partial t}+\frac{\partial \Psi_{1}}{\partial x}=0
$$

A equação (12) garante a preservação do princípio de conservação de massa no processo de difusão. Falta, no entanto, definir o fluxo $\Psi_{1}$. Para os processos clássicos admite-se a lei de Fick, donde:

$$
\Psi_{1}=-D \frac{\partial q}{\partial x}
$$

Onde D é a constante de difusão. Em seu trabalho seminal Einstein [4 mostrou que para partículas difundindo em meio gasoso o coeficiente de difusão é:

$$
D=\frac{k_{B} T}{6 \pi \eta r}
$$

Onde $k_{B}$ é a constate de Boltzmann, $T$ a temperatura $r$ o raio da partícula esférica e $\eta$ a viscosidade do meio. A equação geral da difusão é, portanto:

$$
\frac{\partial q}{\partial t}=\frac{\partial}{\partial x}\left(D \frac{\partial q}{\partial x}\right)
$$

Se $D$ for constante recupera-se a equação (8). A equação diferencial (14) é definida no intervalo $\left[x_{1}, x_{2}\right]$. A solução dessa equação requer a definição de duas condições de contorno impondo uma combinação do potencial $\hat{P}=\hat{P}\left(x_{i}\right)$ e/ou do fluxo $\Psi=\Psi\left(x_{i}\right)$ no contorno $\mathrm{i}=1,2$ para qualquer tempo t. Além disso é necessário conhecer a condição inicial isto é $q(x, 0)=$ $g(x)$ no intervalo $\left[x_{1}, x_{2}\right]$.

Antes de prosseguir e para comparação com soluções na secção seguinte vamos analisar brevemente a solução do problema da difusão no intervalo $x \in[0, a]$ com as seguintes condições de contorno definidas nas extremidades $\mathrm{x}=0$ e $\mathrm{x}=$ a. Para $\mathrm{x}=0$ vamos admitir fluxo nulo, isto é $\Psi_{1}(0, t)=0$ donde $\partial q /\left.\partial x\right|_{x=0}=0$. Na extremidade $\mathrm{x}=\mathrm{a}$ o fluxo é livre. A condição inicial é definida por:

$$
q(x, 0)=q_{0} \cosh (x / a)
$$

Esta função preenche os requisitos impostos pelas condições de contorno, para $\mathrm{x}=0 \partial q /\left.\partial x\right|_{x=0}=\left(q_{0} / a\right)$ $\sinh (0)=0$ e para $\mathrm{x}=\mathrm{a} \partial q /\left.\partial x\right|_{x=a}=\left(q_{0} / a\right) \sinh (1)$

A solução da equação de difusão nesse caso pode ser escrita sob a forma:

$$
q(x, t)=q_{0} f(t) \cosh (x / a)
$$

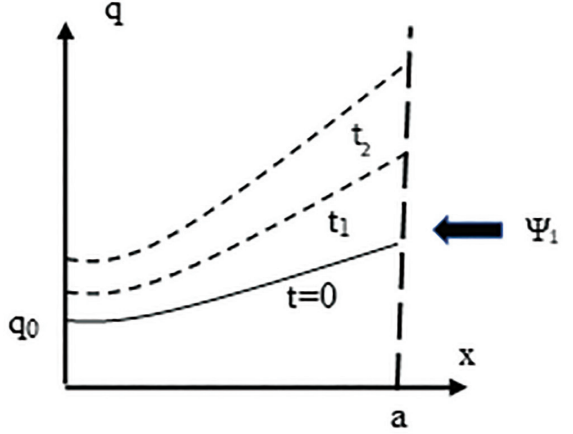

Figura 4: Evolução da concentração segundo a equação (8).

Com a função $f(t)$ a ser determinada. Substituindo essa expressão em (8) obtém-se:

$$
q_{0} \frac{d f}{d t} \cosh \left(\frac{x}{a}\right)=\frac{D}{a^{2}} q_{0} f(t) \cosh \left(\frac{x}{a}\right)
$$

$\mathrm{Ou}$

$$
\frac{d f}{d t}=\frac{D}{a^{2}} f(t)
$$

Donde se segue que

$$
f(t)=A \exp \left(-D t / a^{2}\right) .
$$

Para que a condição inicial seja obedecida é necessário que $A=1$. A solução geral da equação da difusão para as condições definidas acima é:

$$
q(x, t)=q_{0} \exp (\rho t) \cosh (x / a)
$$

Onde $\rho=D / a^{2}$.

A Fig. 4 apresenta esquematicamente a evolução da solução ao longo do eixo x. Note que o fluxo de entrada, isto é, para $\mathrm{x}=$ a é:

$$
\Psi_{1}=-1.1752 D \frac{q_{0}}{a} \exp \left(\frac{D}{a^{2}} t\right)
$$

O fluxo para $\mathrm{x}=\mathrm{a}$ é dirigido para o interior do domínio e portanto a densidade $q(x, \mathrm{t})$ é uma função crescente com o tempo. Haverá aumento exponencial de conteúdo ao longo do tempo.

\section{Processos Difusivos. Fluxo Duplo}

Agora suponha que o processo de redistribuição de partículas seja tal que uma parcela do conteúdo $q_{n}$ da célula $\mathrm{n}$ fique retida temporariamente na própria célula e o restante seja redistribuído para as células vizinhas em partes iguais, conforme mostra a Fig. 5 .

Com esse processo as equações de redistribuição são:

$$
\begin{aligned}
q_{n}^{t} & =(1-\beta) q_{n}^{t-\Delta t}+\frac{1}{2} \beta q_{n-1}^{t-\Delta t}+\frac{1}{2} \beta q_{n+1}^{t-\Delta t} \\
q_{n}^{t+\Delta t} & =(1-\beta) q_{n}^{t}+\frac{1}{2} \beta q_{n-1}^{t}+\frac{1}{2} \beta q_{n+1}^{t}
\end{aligned}
$$




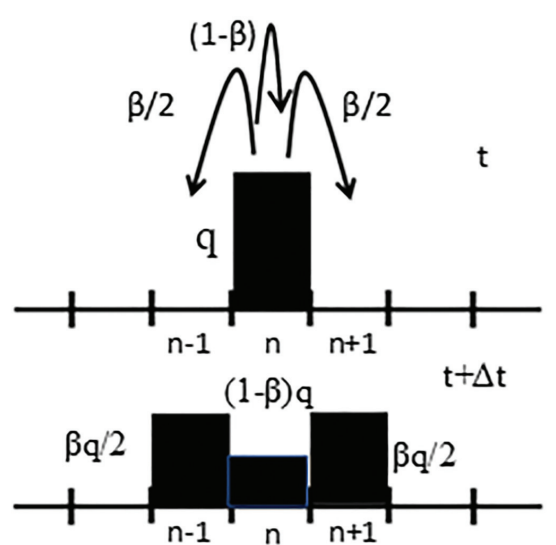

Figura 5: Distribuição simétrica com retenção.

Onde $\beta$ representa a parcela de partículas sendo redistribuídas simetricamente e $(1-\beta)$ a parcela retida temporariamente. Fazendo a diferença entre as duas distribuições obtém-se sucessivamente:

$$
\begin{aligned}
q_{n}^{t+\Delta t}-q_{n}^{t}= & -\beta(1-\beta) q_{n}^{t-\Delta t}+\beta(1-\beta)\left(q_{n-1}^{t-\Delta t}+q_{n+1}^{t-\Delta t}\right) \\
& -\frac{1}{2} \beta\left(q_{n-1}^{t-\Delta t}+q_{n+1}^{t-\Delta t}\right) \\
& +\frac{1}{4} \beta^{2}\left(q_{n-2}^{t-\Delta t}+2 q_{n}^{t-\Delta t}+q_{n+2}^{t-\Delta t}\right) \\
q_{n}^{t+1}-q_{n}^{t}= & \frac{1}{4} \beta\left[\left(q_{n-2}^{t-\Delta t}-2 q_{n-1}^{t-\Delta t}+q_{n}^{t-\Delta t}\right)\right. \\
& \left.+\left(q_{n}^{t-\Delta t}-2 q_{n+1}^{t-\Delta t}+q_{n+2}^{t-\Delta t}\right)\right] \\
& +\frac{1}{4} \beta(1-\beta)\left[-q_{n-2}^{t-\Delta t}+4 q_{n-1}^{t-\Delta t}\right. \\
& \left.-6 q_{n}^{t-\Delta t}+4 q_{n+1}^{t-\Delta t}-q_{n+2}^{t-\Delta t}\right]
\end{aligned}
$$

Em termos de diferenças finitas admitindo-se a variável $q(x, t)$ suficientemente suave $\left(\mathrm{C}^{4}\right.$ em $\mathrm{x}$ e $\mathrm{C}^{1}$ em t) obtemos (Apêndice I):

$$
\Delta q_{n}^{t+\Delta t}=\beta\left\{\frac{1}{2} \Delta^{2} q_{n}^{t-\Delta t}-\frac{1}{4}(1-\beta) \Delta^{4} q_{n}^{t-\Delta t}\right\}
$$

Ou:

$$
\begin{aligned}
\frac{\Delta q_{n}^{t+\Delta t}}{\Delta t}= & \beta\left\{\frac{1}{2} \frac{\Delta x^{2}}{\Delta t} \frac{\Delta^{2} q_{n}^{t-\Delta t}}{\Delta x^{2}}\right. \\
& \left.-\frac{1}{4} \frac{\Delta x^{4}}{\Delta t}(1-\beta) \frac{\Delta^{4} q_{n}^{t-\Delta t}}{\Delta x^{4}}\right\}
\end{aligned}
$$

Tomando o limite quando $\Delta x \rightarrow 0$ e $\Delta t \rightarrow 0$ vem:

$$
\frac{\partial q}{\partial t}=\beta D \frac{\partial^{2} q}{\partial x^{2}}-(1-\beta) \beta R \frac{\partial^{4} q}{\partial x^{4}}
$$

Onde:

$$
\begin{gathered}
\Delta x^{2} / 2 \Delta t \rightarrow D \quad \text { e } \quad \Delta x^{4} / 4 \Delta t \rightarrow R \\
\text { para } \Delta x \rightarrow 0 \quad \text { e } \quad \Delta t \rightarrow 0
\end{gathered}
$$

Para que o parâmetro $R$ tenha sentido é necessário que a posição média da distribuição das partículas pertencentes a esse novo modo varie com a raiz quarta do tempo, isto é. $\Delta x \sim(\Delta t)^{1 / 4}$. Esta hipótese é necessária para que o parâmetro $R$ tenha sentido, $R \neg 0$. Note que são dois processos que se desenvolvem em paralelo com velocidades diferentes. O novo processo, que aparece na derivação da equação (9), evolui mais lentamente do que o processo principal, que obedece à lei de Fick, para $t$ suficientemente grande. Isto significa que as partículas no fluxo secundário tendem a se espalhar mais lentamente coerentemente com as hipóteses iniciais. Podemos em princípio imaginar a ocorrência de um processo de difusão constituído por partículas, que ao longo do tempo decaem de um estado de energia principal para um estado de energia secundário degenerado. No processo de decaimento a entropia cresce e as partículas movimentam-se mais lentamente. O modelo que apresentamos é um bom candidato para simular esse tipo de fenômeno desde que os parâmetros $R$ e $\beta$ sejam funções do tempo o que não introduz grandes dificuldades na solução [5]. A interpretação desses parâmetros está associada à existência de dois potenciais distintos que atuam nos dois grupos de partículas. O primeiro grupo constituído pela parcela $\beta q(x, t)$ espalha-se de acordo com o processo clássico de Fick com o fluxo $\Psi_{1}=\left|\Psi_{1}\right|=$

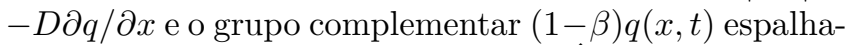
se excitado por um novo potencial $\hat{P}(x)=\beta \partial^{2} q / \partial x^{2}$ que dá origem ao novo fluxo $\Psi_{2}=\left|\Psi_{2}\right|=-R \beta \partial^{3} q / \partial x^{3}$ onde $R$ é um novo parâmetro físico, que designaremos por coeficiente de reatividade.

A equação (9) representa, portanto, um fenômeno complexo que admite a coexistência de dois fluxos evoluindo paralelamente em estados de energia diferentes. O potencial de ativação do fluxo principal de Fick independe da concentração de partículas enquanto o potencial de ativação do fluxo secundário depende da concentração de partículas $\beta$ no estado principal. Em processos que se desenvolvem em compartimentos blindados, processos adiabáticos, o fluxo secundário evolui em um estado de energia ativa inferior ao correspondente ao fluxo primário.

Se admitirmos $\beta=\beta(t)$, a equação 18 representa ainda a transferência de partículas entre os dois fluxos, Fig. 6. Se o processo decair reduzindo a energia ativa presente nas partículas no fluxo principal que migram para o fluxo secundário reduzindo a energia ativa, o processo tende ao repouso. De fato, se $\beta \rightarrow 0$ teremos $\partial q / \partial t \rightarrow 0$. O fluxo secundário não existe por si só, é um subproduto do fluxo primário.

Então a equação 18 representa a variação da densidade de um conjunto de partículas semelhantes divididas em dois conjuntos que evoluem segundo dois fluxos distintos Fig. 6.

$$
\begin{aligned}
& \boldsymbol{\Psi}_{1}=-D \frac{\partial q}{\partial x} \mathbf{n}_{1} \\
& \boldsymbol{\Psi}_{2}=R \beta\left(\frac{\partial^{3} q}{\partial x^{3}}\right) \mathbf{n}_{1}
\end{aligned}
$$




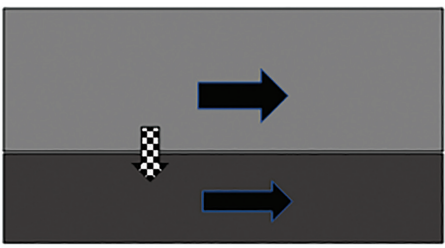

$$
\begin{aligned}
& \text { Fluxo de Fick } \\
& \Psi_{1}=-D \frac{\partial q}{\partial} \mathbf{n} \\
& \text { Fluxo subsidiário } \\
& \Psi_{2}=\beta R \frac{\partial^{3} q}{\partial x^{3}} \mathbf{n} \\
& \Psi_{0} \sim \frac{\partial \beta}{\partial t} \quad \beta=\beta(t) \\
& \begin{array}{l}
\text { Fluxo oculto, interno, } \\
\text { migração entre estados de } \\
\text { energia }
\end{array}
\end{aligned}
$$$$
t_{m}>t_{n}
$$

Figura 6: Difusão com fluxo duplo, $\Psi_{1}$ representa fluxo principal, fluxo de Fick e $\Psi_{2}$ o fluxo secundário, subsidiário ao principal. Pode haver um fluxo interno de transferência de partículas entre os dois estados de energia controlado pelo parâmetro $\beta$.

Sendo que a fração $\beta$ do conjunto $q(x, t)$ é sensível ao potencial correspondente a $\boldsymbol{\Psi}_{1}$ e a fração complementar $(1-\beta)$ é sensível ao potencial correspondente a $\boldsymbol{\Psi}_{2}$. Com essas definições e aplicando o princípio de conservação de massa como apresentado na secção anterior obtém-se a equação geral:

$$
\frac{\partial q}{\partial t}=\frac{\partial}{\partial x}\left(\beta D \frac{\partial q}{\partial x}\right)-\frac{\partial}{\partial x}\left((1-\beta) \beta R \frac{\partial^{3} q}{\partial x^{3}}\right)
$$

Existem teorias que exploram equações de quarta ordem mantendo a hipótese de um fluxo único. As soluções dessas equações têm apresentado dificuldades relativas a princípios de conservação de massa 6. No caso que discutimos acima também podem aparecer problemas semelhantes, mas que são facilmente corrigidos pois as possíveis violações no caso da equação (9) são geradas por incompatibilidade no valor atribuído à fração $\beta$. Isto é a distribuição das partículas entre os dois estados não pode ser arbitrária, o que reforça o sentido físico da teoria. Se for conhecido o processo de migração entre estados correspondentes aos fluxos $\boldsymbol{\Psi}_{1}$ e $\Psi_{2}$ isto é se for conhecida a lei que rege $\beta=\beta(t)$, a mesma equação 18 continua válida. Isto significa que além dos dois processos principais existe ainda um fluxo interno relativo à transferência das partículas de um estado para outro Fig. 6. Desenvolvimento com maiores pormenores podem ser encontrados em [5, 7]. Neste trabalho vamos considerar todas os parâmetros físicos, $D, R$ e $\beta$ constantes. Para o exemplo tratado adiante essa hipótese é suficiente.

Vamos analisar o comportamento da solução da equação 18 definida no mesmo intervalo $x \in\{0, a\}$ e com a mesma condição inicial adotada para o problema clássico discutido acima. Note que como se trata agora de uma equação de quarta ordem em x são necessárias mais duas condições de contorno. Essas condições estão relacionadas ao potencial do fluxo secundário $\hat{P}(x)=\beta \partial^{2} q / \partial x^{2}$ e ao fluxo secundário $\boldsymbol{\Psi}_{2}=-R \beta \partial^{3} q / \partial x^{3} n_{1}$, ambas no contorno $x=0$ e $x=1$. Vamos admitir que para $\mathrm{x}=0$ ambos os fluxos se anulam e que sejam livres em $x=1$. Tomamos para condição inicial como no caso anterior:

$$
q(x, 0)=q_{0} \cosh (x / a)
$$

Com as condições de contorno propostas acima vem:

$$
\begin{array}{lll}
x=0 & \Psi_{1}=\Psi_{2}=0 & \text { fluxo nulo. } \\
x=1 & \Psi_{1} \text { e } \Psi_{2} & \text { fluxos livres, isto é, sem } \\
& & \text { restrições impostas a priori. }
\end{array}
$$

Vamos admitir que a solução seja da forma

$$
q(x, t)=q_{0} f(t) \cosh (x / a)
$$

Como no caso anterior substituindo $q(x, t)$ na equação (18) e após simplificações elementares obtém-se:

$$
\frac{d f}{d t}=\left(\beta \frac{D}{a^{2}} q_{0}-(1-\beta) \beta \frac{R}{a^{4}}\right) f(t)
$$

Donde se segue a solução:

$$
q(x, t)=q_{0} \exp (\rho t) \cosh \left(\frac{x}{a}\right)
$$

O parâmetro $\rho \operatorname{com} r=R / D$ é:

$$
\rho=\frac{D}{a^{2}} \beta\left(1-(1-\beta) \frac{r}{a^{2}}\right)
$$

A diferença essencial entre a solução clássica admitindo o fluxo único e a nova solução admitindo fluxo duplo está na expressão para o parâmetro $\rho$. Para o problema clássico a concentração é sempre crescente, pois $\rho>0$. Para a solução com fluxo duplo $\rho$ pode ser positivo, negativo ou nulo em função dos valores dos parâmetros $\rho$ e $r / a^{2}$ como pode ser facilmente verificado com a Eq. 22. Se $\rho>0$ a concentração é sempre crescente, haverá acúmulo de conteúdo e se $\rho<0$ a concentração reduz-se progressivamente. Se $\rho=0$ haverá estagnação. Fica explicito que a Eq. (22) contempla um leque de soluções muito maior, o que a qualifica para modelar casos mais próximos da realidade.

\section{Aplicação da Teoria de Quarta Ordem Para Simulação de Fluxo de Capital}

Para justificar a apresentação desta proposta é conveniente mostrar que alguns resultados básicos correspondem às expectativas do senso comum para situações reais.

O fluxo de capital é adequado ao nosso propósito pois o movimento de moeda ocorre normalmente em duas direções designados por "receita" e "despesa" ou "venda" e "compra". Portanto, consideraremos o comportamento econômico de uma pequena cadeia social considerando a entrada e saída de capital em um processo bastante 
simples. Análises preliminares mostraram que o uso da teoria de bi-fluxo leva a simulações que reproduzem, pelo menos qualitativamente, os estados econômicos mais importantes.

Considere o caso de uma cadeia econômica no intervalo $(0,1)$. A concentração de recursos, que chamamos de $q(x, t)$, varia entre o ponto inicial $x=0$, com $q(0,0)=0,5$, até o final da cadeia, $x=1, q(1,0)=0,56$ para $t=0$. As condições de contorno em $x=0$ não prescrevem nenhum fluxo e, portanto, o início da cadeia não tem interação com agentes externos. Em $x=1$, os agentes da cadeia interagem com o ambiente externo de modo que o fluxo primário $\boldsymbol{\Psi}_{1}$ seja direcionado para dentro e o fluxo secundário $\boldsymbol{\Psi}_{2}$ para fora, Fig. 7] A solução para este problema com a condição inicial $q(x, 0)=$ $q_{0} \cosh (x / a)$ foi desenvolvida na secção anterior.

Fig. 8 mostra a variação de $\rho \operatorname{com} \beta$ para alguns valores do parâmetro $r$. Claramente, para $\rho>0$, o processo evolui na direção da acumulação de ativos, pois $\exp (\rho t)>1$, o que significa que o volume de entrada de capital excede o volume de saída de capital. Para o caso oposto, $\rho<0$ e $\exp (\rho) t<1$, o processo entra em um ciclo de recessão acumulando perdas progressivamente. Combinações de $\beta$ e $r$ tais que $\rho$ seja igual a zero representam pontos de estagnação que podem ser considerados, em um contexto diferente, pontos de equilíbrio. Preferimos chamar esses pontos de pontos de estagnação, sem ganhos nem perdas.

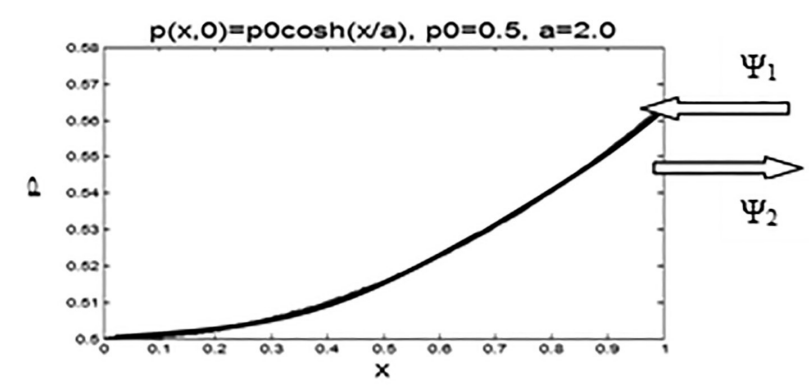

Figura 7: Perfil do fluxo de capital em uma cadeia econômica.

A

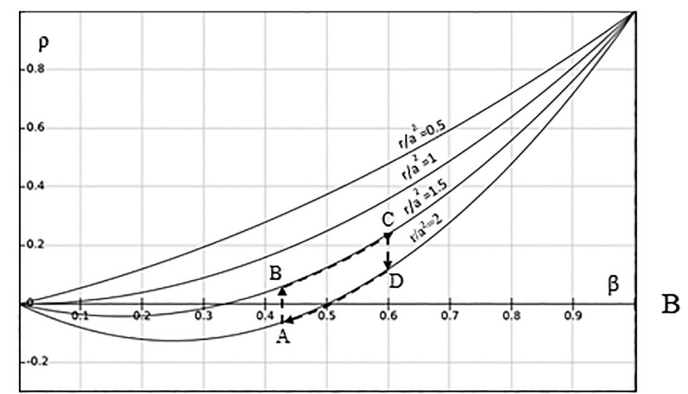

Figura 8: Variação do expoente $\rho$ com $\beta$ correspondendo à fração de entrada de capital para diferentes valores de $r=R / D$. $\mathrm{Na}$ região acima de $\mathrm{AB}$ há crescimento econômico, $\rho>0$. A região abaixo de $A B$ corresponde ao encolhimento econômico $\rho<0$ e os pontos sobre $\mathrm{AB}$ correspondem à estagnação $\rho=0$.
Observe que, conforme posto anteriormente, o fluxo primário $\boldsymbol{\Psi}_{1}$ existe independentemente de $\boldsymbol{\Psi}_{2}$. O fluxo subsidiário $\boldsymbol{\Psi}_{2}$ porém, existe se e somente se $\boldsymbol{\Psi}_{1}$ estiver ativo. Isso significa que empenhar recursos só é possível se houver inicialmente captação de capital. Podemos interpretar os parâmetros $R, D, \beta$ e a como exposto a seguir:

$D$ : representa ferramentas para estimular (desestimular) investimentos. Altos valores de $D$ correspondem à intensificação da taxa de entrada de capitais, representa também a melhoria das exportações ou vendas para o exterior.

$R$ : representa ações para reduzir (aumentar) os gastos. Valores baixos de $R$ correspondem à contenção na taxa de saída de caixa, ou seja, tende a diminuir a taxa de saída de recursos. Pode representar também a substituição de importações com avanço tecnológico interno.

$\beta$ : este é um parâmetro muito importante que regula a distribuição entre o volume de entrada e saída de capital, recebimentos e despesas. Quanto maior o valor de $\beta$ tanto maior a relação venda/compra, (entrada de capital)/(saída de capital). Se $\beta$ for pequeno a taxa de gasto cairá na mesma proporção relativamente à venda.

$a$ : pode ser considerado como a extensão do grupo social ou o conjunto das atividades conectadas que compõem a cadeia produtiva.

Apenas para dar um exemplo, suponha que uma determinada economia siga a trajetória de contração, ou seja, $\rho<0$ representada pelo ponto A, $r / a^{2}=2$ na Fig. 8 Suponha que seja imposto pelo contexto econômicoindustrial que a relação entre entrada de capital e a saída de capital permaneça constante, ou seja, $\beta$ deve ser mantido constante até que a economia apresente uma evolução positiva. Isso seria possível reduzindo o valor de $r=R / D$. Se $D$ for mantido constante porque presumivelmente depende mais de fatores externos fixos, a solução seria reduzir $R$, ou seja, tentar negociar prorrogação dos prazos de pagamento ou estimular o avanço de tecnologias endógenas passando para a curva $r / a^{2}=1.5$. Nesta posição a economia apresenta sinais concretos de expansão e então seria possível atrair novos capitais ou incrementar as vendas, ou seja, aumentar o valor de $\beta$ para chegar ao ponto C. No entanto, se for conveniente aumentar $R$ e acelerar a saída por algum motivo seria possível voltar a $r / a^{2}=2$ e ainda manter a economia no caminho do crescimento, ponto $D$. Note que se o comprador externo quiser influenciar no desempenho interno basta alterar o parâmetro $D$. Se o comprador reduzir o valor de $D$, dificultando a venda, poderá levar a situações de retrocesso pois essa medida tende a aumentar o valor de r. Por outro lado, a facilitação de importações ou o desestimulo da independência tecnológica são fatores desfavoráveis ao progresso na economia. Finalmente é sempre possível 
evitar perdas se for mantida a relação $r / a^{2}<1$ ou $R<$ $D a^{2}$. Isto significa obviamente que se o gasto for inferior ao produto de vendas regulado pelo parâmetro $a$ haverá sempre crescimento com taxas maiores ou menores em função do valor de $\beta$.

Embora haja necessidade de uma análise mais detalhada o exemplo exposto acima mostra que o modelo é promissor particularmente porque admite a afluência simultânea de dois fluxos com a transferência interna entre ambos, um de entrada e outro de saída de capital. Expõe ainda claramente que ações dirigidas no sentido de apurar tecnologias endógenas favorecem o acúmulo de recursos e evitam trajetórias de descapitalização. $\mathrm{O}$ aperfeiçoamento do modelo, além de incluir outros termos que considerem movimentos complementares, deve considerar a variação dos parâmetros ao longo da posição na cadeia assim como no tempo. Isto é, o modelo admite um grande leque de desenvolvimento analítico que o tornem mais próximo de situações reais. Modelos dessa mesma natureza [3] tem sido usados para prever situações de fluxo de capitais mas considerando apenas fluxo em uma direção o que impõe limitações nas aplicações.

\section{Conclusões}

A teoria que admite fluxo duplo conforme exposta acima é mais completa do que a teoria que admite fluxo único. Conforme examinado no item anterior a modelagem de fluxo de capitais é mais bem representada com a hipótese de fluxo duplo. Problemas de dinâmica populacional também podem ser mais bem solucionados com o modelo apresentado nesse texto. Note que a nova equação abre outras possibilidades de modelagem para o tratamento da difusão em meios que são parcialmente homogéneos. De fato os parâmetros de difusão $D$ e de reatividade $R$, são independentes. Um meio pode ser homogêneo quanto à difusão primária, de Fick, e, portanto, com $D$ constante e não homogéneo quanto a difusão secundária, isto é, com $R=R(x)$. Com isso pode-se modelar processos em que as partículas em movimento respondem a estímulos externos localizados, como a presença de atractores.

Um problema que possivelmente pode ser tratado com maior confiabilidade usando a abordagem de fluxo duplo é a evolução de pandemias, como a COVID-19. A transferência do modo infetado para o modo recuperado representando duas correntes paralelas pode possivelmente ser estudada com o modelo de fluxo duplo produzindo resultados mais próximos da realidade.

A teoria exposta acima foi desenvolvida para processos de redistribuição em uma cadeia discreta definida em um espaço unidimensional. A teoria pode ser expandida para processos não homogéneos que evoluem em domínios bidimensionais, isto é, $D=D(x, y), R=R(x, y)$ e $\beta=\beta(x, y)$ 4. Essa opção torna o modelo bastante flexível para aplicações. Se um conjunto de células estiver concentrado em uma região afastada da posição onde há disponibilidade de nutrientes o modelo mostra que há um deslocamento inicial na direção do nutriente que precede o movimento previsto de difusão.

Estudos recentes, de células que se deslocam com mudança de estado, têm sido desenvolvidos com processos difusivos admitindo fluxo simples com o coeficiente de difusão função do tempo, $D=D(t)$. Isto é, a taxa de difusão varia com o tempo, admitindo-se que todas as células mudam de estado simultaneamente, o que é uma hipótese bastante limitada [8].

Para processos físico-químicos o modelo aplica-se a fenómenos de difusão de partículas da mesma natureza que se movem em estados de energia diferentes. Tratase, pois, de processo de difusão em multiestados cada um com sua respetiva energia ativa. O modelo admite, que no estado principal, difusão de Fick, a entropia está próxima a zero e cresce a medida que as partículas se transferem para o fluxo secundário. Quando se extinguem as partículas no estado principal o sistema fica congelado e não há mais movimento, $q(x, t)=$ constante. $\mathrm{Na}$ realidade o modelo inclui indiretamente a degeneração de energia ativa nos processos de difusão.

As principais propostas de modelos para a difusão foram desenvolvidas no início do século passado [4, 9, 10]. Salinas 11] apresenta um histórico bastante completo da evolução das teorias propostas para modelar processos de difusão. Esperamos que este texto e outros que têm sido publicados tratando sobre esse tema possam despertar o interesse de estudantes e pesquisadores tanto na área de modelagem como na área experimental. É muito difícil introduzir novas teorias em temas bastante consolidados no cenário científico internacional. Desviar do "mainstream", do convencional, é difícil, mas compensador no final das contas. Infelizmente o requisito de volume de publicações, impostos nos nossos dias, afasta jovens pesquisadores de aventuras intelectuais tão importantes para o progresso da ciência. De qualquer modo espero que esse texto estimule alguns jovens a arriscar parte de seu tempo para explorar novos caminhos, seja na linha teórica, seja na linha experimental.

\section{Desafio}

Considere o caso de retenção com redistribuição assimétrica $\left|\gamma_{1}\right| \neq\left|\gamma_{2}\right|$, Fig. 9 Se a fração de retenção for igual

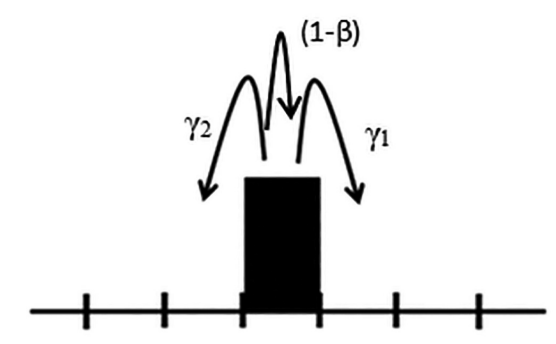

Figura 9: Retenção com distribuição assimétrica. Note $\gamma_{1}>$ $0 \gamma_{2}<0$ 
a $(1-\beta)$ tem-se $\left|\gamma_{1}\right|+\left|\gamma_{2}\right|=\beta$. Neste caso pode-se esperar que a equação resultante do tratamento discreto seja da forma:

$$
\frac{\partial q}{\partial t}=A_{0} q+A_{1} \frac{\partial q}{\partial x}+A_{2} \frac{\partial^{2} q}{\partial x^{2}}+A_{3} \frac{\partial^{3} q}{\partial x^{3}}+A_{4} \frac{\partial^{4} q}{\partial x^{4}}
$$

Para o caso sem retenção, $\beta=1$, usando a formulação discreta obtém-se $A_{0}=A_{3}=A_{4}=0$, como deve ser, pois nesse caso temos o processo de advecção ou transporte superposto ao processo de difusão [12]. Mas para o caso geral de redistribuição assimétrica com retenção, $\beta<1$, espera-se que $A_{0} \neq A_{3} \neq A_{4} \neq 0$. Portanto trata-se este desafio da determinação dos parâmetros $A_{i}$ $\mathrm{i}=0,4$ na equação acima.

\section{Material suplementar}

O seguinte material suplementar está disponível online: Apêndice I - Formulação discreta de operações de diferenciação.

\section{Referências}

[1] A. Fick, Annalen der Physic 94, 59 (1855).

[2] R. Brown, Philosophical Magazine 4, 161 (1827).

[3] F. Black e S. Myron, Journal of Political Economy 81, 637 (1973).

[4] A. Einstein, Annalen der Physik 322, 549 (1905).

[5] M. Jiang, The Fourth Order Diffusion Model for a BiFlux Mass Transfer. Tese de Doutorado, Universidade Federal do Rio de Janeiro, Rio de Janeiro (2017).

[6] P. Broadbridge, Entropy 10, 365 (2008).

[7] L. Bevilacqua, M. Jiang, A.J. Silva Neto e A.C.R.N. Galeão, J Braz. Soc. Mech. Sci. Eng. 38, 1421 (2016).

[8] C. Riedel, R. Gabizon, C.A.M Wilson, K. Hamadani, K. Tsekouras, S. Marqusee, S.Pressé e C. Bustamente, Nature 517, 227 (2015).

[9] M. Smoluchowski, Annalen der Physik 326, 756 (1906).

[10] P. Langevin, C. R. Acad. Sci. Paris 146, 530 (1908).

[11] S. Salinas, Rev. Bras. Ensino Fís. 27, 263 (2005).

[12] L. Bevilacqua, A.C.N. Galeão e F. Pieronbom, J Braz. Soc. Mech. Sci. Eng 34, 166 (2011). 